\title{
Analysis of the Levels of Endothelin, Homocysteine and Blood Lipid in Patients with Clinical and Subclinical Hyperthyroidism
}

\author{
Xu Qian ${ }^{1,2}$, Zhang Jing-yu ${ }^{3}$, Li Hong-yan ${ }^{1,2, ~ *, ~ H o u ~ J i a n-z h a n g ~}{ }^{4}$ \\ ${ }^{1}$ Department of Medical Technology of Cangzhou Medical College, Cangzhou, China \\ ${ }^{2}$ Cangzhou Thyroid Disease Engineering Research Center, Cangzhou, China \\ ${ }^{3}$ Laboratory Department of Cangzhou Hospital Integrated of Chinese and Western Medicine, Cangzhou, China \\ ${ }^{4}$ Cangzhou People's Hospital, Cangzhou, China
}

Email address:

czyzjyk@126.com (Li Hong-yan),1925588501@qq.com (Xu Qian)

${ }^{*}$ Corresponding author

\section{To cite this article:}

Xu Qian, Zhang Jing-yu, Li Hong-yan, Hou Jian-zhang. Analysis of the Levels of Endothelin, Homocysteine and Blood Lipid in Patients with Clinical and Subclinical Hyperthyroidism. American Journal of Life Sciences. Vol. 7, No. 1, 2019, pp. 27-30. doi: 10.11648/j.ajls.20190701.15

Received: February 26, 2019; Accepted: April 17, 2019; Published: April 28, 2019

\begin{abstract}
Objective] To discuss the changes of endothelin (ET), homocysteine (Hcy) and blood lipids levels in patients with hyperthyroidism and subclinical hyperthyroidism, and to explore their clinical significance. [Methods] The authors choose 251 cases of hyperthyroidism, 242 cases of subclinical hyperthyroidism and 130 cases of the control group to test ET, Hcy, TC, TG, HDL, LDL and LPa. [Results] The level of ET of Hyperthyroidism group is higher than subclinical hyperthyroidism group and the control group. The level of ET of subclinical Hyperthyroidism group is higher than the control group $(\mathrm{P}<0.05)$. The level of Hcy of Hyperthyroidism group and subclinical Hyperthyroidism group was lower than the control group $(\mathrm{P}<0.05)$. The level of TG, TC, LDL, LPa of subclinical Hyperthyroidism group are lower than the control group $(\mathrm{P}<0.05)$. The level of TG, TC, HDL, LDL, LPa of Hyperthyroidism group are lower than the control group and subclinical hyperthyroidism group $(\mathrm{P}<0.05)$. [Conclusions] All of ET, Hcy and lipids participate in the occurrence and development process of hyperthyroidism and subclinical hyperthyroidism. Monitoring the change of related indexes is helpful to predict the transformation of subclinical hyperthyroidism to hyperthyroidism and the occurrence of cardiovascular disease.
\end{abstract}

Keywords: Hyperthyroidism, Subclinical Hyperthyroidism, Hcy, ET, Blood Lipids

\section{Introduction}

Hyperthyroidism is one of the most common endocrine diseases in clinic. It is an autoimmune disease caused by excessive secretion of thyroid hormone in the body; it leads to the multi-system syndrome, which the increase of excitability of nervous, digestive, circulatory system and hypermetabolism is the main clinical manifestations. If not treated in time, it may cause metabolic disorders, atherosclerosis and hyperthyroidism heart disease [1], hyperthyroidism myopathy, hyperthyroidism ophthalmopathy, hyperthyroidism periodic paralysis and even hyperthyroidism crisis and other complications, more commonly found in cardiovascular and nervous system [2]. Subclinical hyperthyroidism refers to specific types of hyperthyroidism that the normal range of serum between FT3 and FT4, but the serum thyrotropin (TSH) is lower than the reference range. Patients, often due to no clinical symptoms, or symptoms are not accurate or not specific, are easy to miss diagnose and to be misdiagnosed, thus, effective treatment are not taken in time, and subclinical hyperthyroidism developed into clinical hyperthyroidism [3]. ET is not only an important factor in regulating cardiovascular function [4], but also is involved in neuroendocrine regulation. Hcy is an independent risk factor for cardiovascular and cerebrovascular diseases. Thyroid dysfunction often leads to abnormal lipid metabolism. This study analyzed the changes of ET, Hcy and blood lipid levels in patients with clinical hyperthyroidism and subclinical 
hyperthyroidism in order to explore their relationship with the occurrence and development of clinical hyperthyroidism and subclinical hyperthyroidism, and to provide objective basis for the prevention and treatment of clinical hyperthyroidism and subclinical hyperthyroidism and related diseases.

\section{Datas and Methods}

\subsection{General Datas}

From March 2016 to October 2018, 493 patients (194 males and 299 females) were selected from the Department of Endocrinology, Cangzhou Hospital Integrated of Chinese and Western medicine, Hebei Province, among which, 251 persons are clinical hyperthyroidism patients (hyperthyroidism group, 98 males, 153 females, age 21 70 (42.2 \pm 15.1$)$ years old) and 242 persons are subclinical hyperthyroidism patients (subclinical hyperthyroidism group, 95 males, 147 females, age $21 \sim 70(46.5 \pm 17.4)$ years old). At the same time, 130 healthy persons (control group, 58 males, 72 females, age $21 \sim 70(45.3 \pm 12.5)$ years old $)$ were selected. There was no significant difference in sex and age among the three groups $(\mathrm{P}>0.05)$.

\subsection{Methods}

After receiving a fasting hemospasia in the early morning, venous blood $(4 \mathrm{ml})$ was collected from the three groups, resting for 1 to 2 hours at room temperature and then serum was separated by high-speed centrifugation. The five indexes of serum thyroid function: free triiodothyronine (FT3), free thyroxine (FT4), total triiodothyronine (TT3), total thyroxine (TT4) and thyrotropin (TSH) were determined by Cobas e602 Automatic Immune Diagnostic Assay System, the reagents are all Roche's specialized supporting products. ET was determined by Elisa kit from Shanghai Kanu Biotech Co. Ltd; Hcy was determined by Abbott Laboratories AXSYM automatic immune analyzer and the chemiluminescence method; Serum TG, TC, HDL, LDL and LPa were determined by HITACHI-020 automatic biochemical analyzer, the reagents are from Heguang Co. Ltd of Japan.

\subsection{Diagnostic Standard}

Diagnostic standard based on the 2008 Chinese guidelines for diagnosis and treatment of thyroid Diseases: (1) Clinical hyperthyroidism: have symptoms or signs of hyperthyroidism, and conforms with $\mathrm{TSH}<0.27 \mathrm{mIU} / \mathrm{L}, \quad \mathrm{FT} 3>7.1 \mathrm{pmol} / \mathrm{L}$, FT4 $>22 \mathrm{pmol} / \mathrm{L}$; (2) Subclinical hyperthyroidism: conforms with $\mathrm{TSH}<0.27 \mathrm{~m} \mathrm{IU} \mathrm{/} \mathrm{L,} \mathrm{and} \mathrm{FT3} \mathrm{as} \mathrm{well} \mathrm{as} \mathrm{FT4,} \mathrm{were} \mathrm{within}$ the normal range.

\subsection{Statistical Analysis}

SPSS19.0 statistical software was used to process the data, the metrological data were expressed as $\bar{x} \pm \mathrm{s}$, the quantitative data were compared by t-test, and the correlation among the factors was analyzed by linear correlation analysis. $\mathrm{P}<0.05$ suggests that there were significant differences.

\section{Results}

\subsection{Comparison of the Levels of ET and Hcy in Each Group}

The level of ET in hyperthyroidism group was higher than that in Subclinical hyperthyroidism group and control group, the difference was statistically significant $(\mathrm{P}<0.05)$. The level of ET in Subclinical hyperthyroidism group was higher than that in control group, and the difference was statistically significant $(\mathrm{P}<0.05)$. Both the level of Hcy in hyperthyroidism group and subclinical hyperthyroidism group were lower than that in control group, the difference was statistically significant $(\mathrm{P}<0.05)$. There was no significant difference in Hcy level between hyperthyroidism group and Subclinical hyperthyroidism group $(\mathrm{P}>0.05)$. The results are shown in Table 1.

Table 1. Comparison of the levels of ET and Hcy in each group ( $x \pm s)$.

\begin{tabular}{llll}
\hline results & Subclinical hyperthyroidism group $(\mathbf{n}=\mathbf{2 4 2})$ & hyperthyroidism group $(\mathbf{n}=\mathbf{2 5 1})$ & control group $(\mathbf{n}=\mathbf{1 3 0})$ \\
\hline $\mathrm{ET}(\mathrm{ng} / \mathrm{L})$ & $126.13 \pm 17.61$ & $137.28 \pm 14.57$ & $110.19 \pm 12.62$ \\
$\mathrm{Hcy}(\mathrm{umol} / \mathrm{L})$ & $11.21 \pm 2.81$ & $10.69 \pm 2.17$ & $13.85 \pm 1.92$ \\
\hline
\end{tabular}

\subsection{Comparison of Blood Lipid Levels in Each Group}

TG, TC, HDL, LDL, LPa in hyperthyroidism group was lower than those in control group and Subclinical hyperthyroidism group, the difference was statistically significant. $(\mathrm{P}<0.05) \mathrm{TG}, \mathrm{TC}, \mathrm{HDL}, \mathrm{LDL}, \mathrm{LPa}$ in Subclinical hyperthyroidism group were lower than those in control group, except HDL, the difference was statistically significant $(\mathrm{P}<0.05)$. The results are shown in Table 2 .

Table 2. Comparison of blood lipid levels in each group ( $x \pm s)$.

\begin{tabular}{llll}
\hline results & Subclinical hyperthyroidism group $(\mathbf{n}=\mathbf{2 4 2})$ & hyperthyroidism group $(\mathbf{n}=\mathbf{2 5 1})$ & control group $(\mathbf{n = 1 3 0})$ \\
\hline $\mathrm{TG}(\mathrm{mmol} / \mathrm{L})$ & $1.24 \pm 0.52$ & $1.06 \pm 0.70$ & $1.44 \pm 0.47$ \\
$\mathrm{TC}(\mathrm{mmol} / \mathrm{L})$ & $3.78 \pm 1.15$ & $3.07 \pm 1.41$ & $4.51 \pm 0.92$ \\
$\mathrm{HDL}(\mathrm{mmol} / \mathrm{L})$ & $1.38 \pm 0.41$ & $1.25 \pm 0.19$ & $1.41 \pm 0.25$ \\
$\mathrm{LDL}(\mathrm{mmol} / \mathrm{L})$ & $2.76 \pm 0.91$ & $2.49 \pm 1.12$ & $2.97 \pm 1.01$ \\
$\mathrm{LPa}(\mathrm{mg} / \mathrm{L})$ & $212.16 \pm 18.73$ & $197.16 \pm 25.18$ & $220.37 \pm 21.46$ \\
\hline
\end{tabular}




\section{Discussions}

ET is a small Micro molecule vasoactive peptide synthesized and secreted by vascular endothelial cells. It has a strong and persistent affection of vasoconstriction and spasticity [5], it is a powerful vasoconstrictor factor [6], and plays an important role in maintaining basal vascular tension and homeostasis of the cardiovascular system [7]. Its overexpression can induce myocardial smooth muscle cell to lysis and apoptosis, Myocardial fibrosis, and vasospasm [8]. A bunch of people represented by Chai Guolu et al [9] reported that the level of ET in patients with hyperthyroidism was significantly higher than that in normal people. People like Chen Xuehui et al [8] found that thyroid hormone and ET have feedback and negative feedback mechanism. The pathogenesis of hyperthyroid heart disease is closely related to IL-1, TNF- $\alpha$ and ET. It was found by my research that the level of ET in the hyperthyroidism group was higher than that in the subclinical hyperthyroidism group and the control group, and the level of ET in the Subclinical hyperthyroidism group was higher than that in the control group. The difference was statistically significant $(\mathrm{P}<0.05)$. It is a suggestion that ET may be involved in the occurrence and development of subclinical hyperthyroidism and clinical hyperthyroidism, and may play an important role in predicting whether subclinical hyperthyroidism develops into clinical hyperthyroidism and may have caused the occurrence of related complications.

Hcy is a sulfur-containing amino acid that presents in very small amount in a human body. Hcy is closely associated with some common clinical diseases, such as cardiovascular disease, nervous system disease, kidney disease, diabetes mellitus, liver disease and so on, among which, Cardiovascular system and nervous system disease are more likely to be found [10]. Through spontaneous oxidation, Hcy produces peroxides and superoxides, which damage vascular endothelial cells and stimulate the excessive growth of vascular smooth muscle, causing the accumulation and precipitation of plasma lipoprotein, thus accelerating the process of atherosclerosis. Mao Lei et al [11] confirmed that high Hcy is one of the risk factors of acute coronary syndrome. Liu Xiangru et al [12] found that the level of plasma Hcy in type 2 diabetic patients was significantly higher than that in healthy groups. The level of serum Hcy may also be related to cognitive function impairment $[13,14]$. In our research, we found that the levels of Hcy in group hyperthyroidism and subclinical hyperthyroidism group were both lower than those in the control group, this consistent with reported on the literature $[15,16]$. Therefore, it is suggested that the detection of serum Hcy level may be helpful to the judgment of thyroid function [17].

The whole body of a Hyperthyroidism patient is in the state of a high metabolic level, the utilization of fat and protein increased, and thus decreased the fat reserve, serum TG and TC. My research shows that the TG, TC, HDL, LDL, LPa of the hyperthyroidism group is lower than the control group; the difference is statistically significant, this consistent with many scholars reports. [18-20]. Our research also showed that TG, TC,
HDL, LDL, LPa in hyperthyroidism group were lower than those in subclinical hyperthyroidism group, and those in the subclinical hyperthyroidism group lower than those in control group, except HDL, the difference was statistically significant, suggesting that subclinical hyperthyroidism patients may not having clinical symptoms, but the metabolic disorder of blood lipid has already begun, this, if without being effectively treated, may further aggravate the metabolic disorder state of blood lipid, which is a risky factor of cardiovascular and cerebrovascular diseases [21]. Therefore, it is necessary to strengthen the monitoring of serum lipids in patients with hyperthyroidism and subclinical hyperthyroidism in order to intervene and treat the disease early [22].

\section{Conclusion}

Briefly, ET, Hcy and blood lipid are all involved in the occurrence and development of clinical hyperthyroidism and subclinical hyperthyroidism, and a close monitor on the changes of related indexes would be helpful for disease evaluation and early prediction of the transformation from subclinical hyperthyroidism to clinical hyperthyroidism and the occurrence of cardiovascular and cerebrovascular diseases.

\section{Fund Project}

Self-financed Project of Hebei Science and Technology Research and Development Program (No. 162777205).

Cangzhou Science and Technology Research and Development Guidance Program Project (Ref. 151302011).

\section{References}

[1] Su Jun-ping, Zhang-Bo, Liu Chun-yan, et al. Changes in levels of thyroid hormone osteometabolism-related cytokines and bone metabolism-related indicators in hyperthyroidism patients and their clinical significance [J]. Shanxi Medical Journal, 2017, 46 (4):413-415.

[2] Hou Zhen-jiang, Zhang Jing-yu, Bian Yun-zhuo, et al. Changes of serum lipid level, its ratio and cystatin $\mathrm{C}$ level in patients with hyperthyroidism and subclinical hyperthyroidism [J]. Shandong Medical Journal, 2017, 57(39):91-93.

[3] Chen-Hua, Xin-Ning. Clinical efficacy and changes in serum lipid index of levothyroxine in subclinical and clinical hypothyroidism [J]. Chinese Journal of Clinical Research, 2014, 6 (9):84.

[4] Guo Ya-qing, Wei Xi-liang, Zhao Dan-ning, et al. Plasma endothelin and carbon monoxide levels in patients with subclinical hypothyroidism [J]. China Journal of Modern Medicine, 2011, 21 (11):1407-1409.

[5] Zhou Yan-guo, Mai Rui-lin, Huang Min-xuan, et al. Effects of alprostol on plasma endothelin, homocysteine and coagulation function in patients with coronary heart disease [J]. Hainan Medical Journal, 2018, 29(07):917-919.

[6] Pancotto TE, Rossmeisl JH, Huckle WR, et al. Evaluation of endothelin-1 and MMPs-2, -9, -14 in cerebrospinai fluid as indirect indicators of blood-brain barrier dysfunction in chronic canine hypothyroidism [J]. Res Vet Sci, 2016, 105:115-120. 
[7] Li Ying-hui, Li-Jun, Ren Si-ying, et al. Effects of rosiglitazone on the expression of endothelin-1 around the lesion and the permeability of blood-brain barrier in rabbits with cerebral hemorrhage [J]. Neural Injury and Functional Reconstruction, 2018, 13(03):109-112.

[8] Chen Xue-hui, Yin Qing-feng, Wang-Tao, et al. Changes of important plasma factors in radionuclide iodine therapy for hyperthyroidism heart disease [J]. Medical Recapitulate, 2013, 19(13):2492-2494.

[9] Chai Guo-lu, Tan Li-yan, Yang Yu-hong, et al. Changes of plasma endothelin and nitric oxide levels in patients with hyperthyroidism and their clinical significance [J]. Heilongjiang Medicine and Pharmacy, 2004(04):34-35.

[10] Hu Yao-shi, Zeng Zhi-yu. Research Progress on the Relationship between Homocysteine and Common Clinical Diseases [J]. Chinese Journal of New Clinical Medicine, 2017, 10(08):811-815.

[11] Mao-Lei, Geng Guo-ying, Zhao Ming-hu, et al. Effects of rosuvastatin on serum levels of high-sensitivity C-reactive protein and homocysteine in patients with acute coronary syndrome [J]. Clinical Research, 2018(06):84-85.

[12] Liu Xiang-ru, Li-Yue, Yang-Bin, et al. Analysis of plasma homocysteine level and related factors in patients with type 1 and type 2 diabetes mellitus [J]. Contemporary Chinese Medicine, 2018(14):1-2.

[13] Min JY, Min KB. The Folate-Vitamin B12 Interaction, Low Hemoglobin, and the Mortality Risk from Alzheimer's Disease [J]. J Alzheimers Dis, 2016, 52 (2):705-712.

[14] Wang Xiao-quan, Wang Zu-sen, Shen Yu-hua, et al. Study on the correlation between serum homocysteine level and P300 in late-onset depression [J]. Journal of Psychiatry, 2017, 30(06): 410-412.
[15] Pan Da-fu, Zhang Li-juan, Ren Yue-zhong. Study on plasma homocysteine level and MTHFR gene polymorphism in patients with hyperthyroidism and hypothyroidism [J]. Chinese Journal of Endocrinology and Metabolism, 2004(02):36-37.

[16] Dai Li-li, Liu Jia-jia, Mi Yu-jing et al, Study on the correlation between subclinical thyroid dysfunction and serum resistin and homocysteine [J]. Chinese Journal of Health laboratory Technology, 2015, 25(14):2346-2348.

[17] Wu Zai-rong, Zhang Li-rong, Pan Yu-qin, et al. Clinical significance of serum homocysteine and lipid levels in patients with thyroid dysfunction [J]. Progress in Modern Biomedicine, 2009, 9 (22): 4276-4278.

[18] Xiao-Lin, Guo-Mei, Fan Ruiyun. The relationship between thyroid hormone levels and lipid metabolism in hyperthyroidism $[\mathrm{J}]$. Chinese Journal of Laboratory diagnosis, 2016, 20(10):1679-1681.

[19] Tan-Qian. Clinical analysis of abnormal metabolism of blood sugar and lipid in patients with hyperthyroidism and hypothyroidism [J]. Capital Food Medicine, 2018, 25(09):19.

[20] Yuan-Zhuo. Metabolism of blood lipid and blood sugar in patients with abnormal thyroid function during pregnancy $[\mathrm{J}]$. Maternal and Child Health Care of China, 2017, 32 (9):1874-1876.

[21] Gu-Qing, Shao Ke-ke, Wang Yun-xia. Analysis of lipid and serum protein levels in patients with thyroid dysfunction [J]. Laboratory Medicine and Clinic, 2014, 11(24):3455-3456.

[22] Duan Wen-bing, Liu-Yun, Zhang Lu-lu, et al. Correlation analysis of blood lipid, HCY, blood routine and indexes of thyroid function in patients with subclinical thyroid dysfunction [J]. Journal of New Medicine, 2018, 49 (1): 42-46. 